\title{
An ATM-Based Intelligent Optical Backplane Using CMOS-SEED Smart Pixel Arrays and Free-Space Optical Interconnect Modules
}

\author{
Dominic J. Goodwill, Kent E. Devenport, and H. Scott Hinton, Senior Member, IEEE
}

\begin{abstract}
The architecture, smart pixel array chip design, and optical design of an intelligent free-space digital optical backplane for ATM switching are presented. The smart pixel chip uses reflective SEED (self-electrooptic effect device) optical modulators and detectors flip-chip bonded to CMOS circuitry. This chip is one of the most complex designs ever reported in this technology, and it operates at a simulated backplane clock rate of $125 \mathrm{MHz}$. The low-loss optical system employs $f / 4$ diffractive minilenses and microlenses to interconnect clusters of smart pixels, and it is shown to allow 2060 connections per chip if $1-\mathrm{cm}^{2}$-sized smart pixel chips are used. This gives a predicted bisection bandwidth of around $1 \mathrm{~Tb} / \mathrm{s}$ across a 10-in circuit board edge for a full-sized system.
\end{abstract}

\section{INTRODUCTION}

$\mathbf{O}$ $\mathrm{NE}$ of the practical limiting factors in the development and acceptance of large ATM switching systems (as well as teraflop multiprocessor computing systems) is packaging. Currently, large systems (64 processing nodes and above) can require multiple cabinets to house all their electronic equipment. This "low density packaging" not only leads to expensive systems but also suffers from increased latency, skew, and other physical problems that limit overall performance. As thermal management schemes have progressed, there is a growing trend to package more electronics into smaller physical volumes. The result is the integration of more processing nodes per integrated circuit, more processing nodes per printed circuit board (PCB), and more printed circuit boards per shelf. This necessary hardware compression leads to an interconnect bottleneck at the backplane.

One approach to overcome these interconnection limitations of electrical backplanes is to exploit the temporal and spatial bandwidth available with free-space optical technology. A free-space optical backplane is composed of a large number of digital optical communication channels, created by simple optical connections between smart pixel arrays (SPA's) on successive PCB's. These SPA's are optoelectronic devices, consisting of optical inputs and/or outputs and electronic processing circuitry. Data to be transferred between PCB's is injected into the optical communication channels via the

Manuscript received March 15, 1996; revised July 9, 1996. This work was supported by the Hudson Moore, Jr. Chair at the University of Colorado in Boulder. The work of K. E. Devenport was supported by a fellowship from the Optoelectronic Computing Systems Center, funded by the NSF.

The authors are with the University of Colorado, Department of Electrical and Computer Engineering, Boulder, CO 80309-0425 USA.

Publisher Item Identifier S 1077-260X(96)07976-2.
SPA's and is then transmitted to the destination PCB's where the SPA's extract the optical signals and convert them back into electrical form. The potential connectivity of these optical backplanes includes the ability to provide over 10000 highperformance connections per PCB while supporting bisection bandwidths in excess of $1 \mathrm{~Tb} / \mathrm{s}$. Perhaps more important than this raw connectivity is the ability for intelligence to be added to each of the optical communication channels through the electronic circuitry in the SPA's. Intelligence can be readily embedded in a smart pixel system due to the very close proximity of individual processing elements (in our case, CMOS logic) with their corresponding set of optical inputs and outputs. It is this intelligence, and its effective use, that is the focus of current research on "intelligent optical backplanes."

In this paper, we focus primarily on the SPA design, the optoelectronic interface, and the optical hardware design for a free-space optical backplane which implements a HyperPlanebased [1], [2] ATM switching fabric. We first outline the architecture of our parallel ATM switching fabric in Section II, showing how buffering and embedded control lead to enhancements in the overall system performance. The circuit design and operation of the corresponding SPA chip is described in Section III, and in Section IV, the particular characteristics of the sensitive, high-speed optical receivers and modulator drivers are discussed. A set of physical optomechanical constraints is then presented, leading to the description of a modular set of optics in Section $V$ which are suitable for interconnecting the SPA chip.

\section{A HyperPlane-Based ATM Switch}

One approach to building an internally nonblocking ATM switching fabric is shown in Fig. 1. For this type of switching fabric, it is assumed that the users will independently send SONET streams containing ATM cells to be switched by the fabric. The users are not shown in the figure, but their connection is represented by the optical fibers at the bottom of the figure. The boxes labeled "switching nodes" have the responsibility of interpreting the arriving ATM cells and generating a fabric address to be prepended to the beginning of each cell. These prepended addresses will be used to direct the cells through the switching fabric. The ATM cells will also be converted from a serial data stream to a parallel stream $n$ bits wide. These switching node functions are provided by off-theshelf electronic integrated circuits. After the fabric address has 


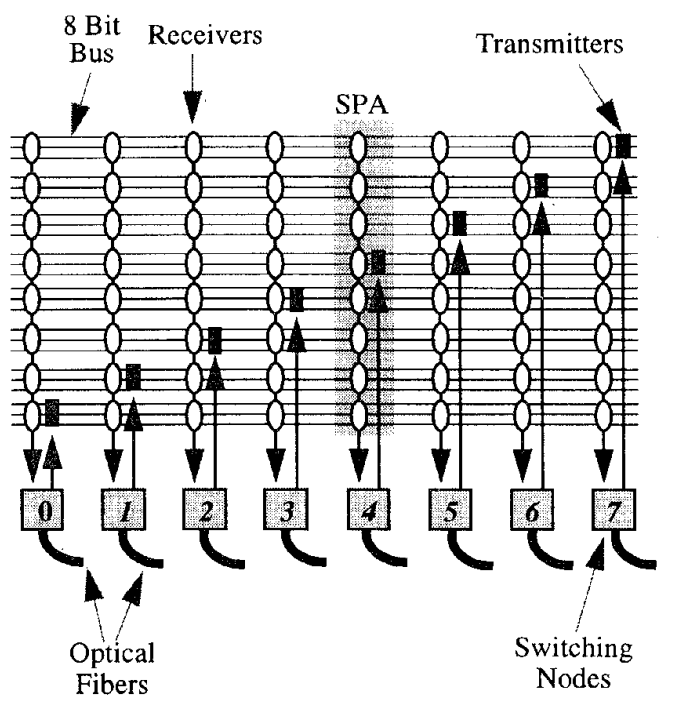

Fig. 1. Bus-based ATM switching fabric.

been prepended to an entering cell, the partitioned $n$-bit words will be injected into the HyperPlane through an $n$-bit-wide node channel. In the HyperPlane, a fixed transmitter assignment is configured at start-up, such that only one switching node is permitted to transmit data on a given node channel. Concurrently, all the receivers are continually listening for their address on all the parallel node channels simultaneously. When a receiver detects its address on a node channel, it extracts the cell from the HyperPlane and directs it to the destination switching node. The cell is then directed via the output fiber to the destination user.

The system implemented in this paper utilizes this basic architecture by: 1) integrating all the required electronics for each switching node onto a single printed circuit board, 2) integrating all the receivers and transmitters required for each switching node into a single SPA, and 3) interconnecting all the SPA's with point-to-point optical interconnects. Note that this is an ideal architecture for a SPA-based switching fabric since the optical bandwidth of the SPA's is $\mathrm{O}(P \bullet N \bullet B)$, while the electrical bandwidth from each PCB to its SPA is only $\mathrm{O}(N \bullet B)$, where $P$ is the number of PCB's, $N$ is the number of users on each $\mathrm{PCB}$, and $B$ is the bit rate of each user.

We now present the protocols for ATM cell based communication on the HyperPlane. When an $(n+1)$-bit node channel of a given SPA is configured to send ATM cells, the protocol is as follows.

1) ATM cells are injected one word ( $n$ bits) at a time.

2) The first word ( $n$ bits) of each ATM cell transmission consists of the prepended fabric header, with the $(n+$ 1)th bit set to 1 to indicate that this is a header. This header contains the destination address of the accompanying cell data. For all words which are not headers, the $(n+1)$ th bit is set to 0 .

3) Idle data (the all-zero string) is sent when no ATM cell is available for transmission.
When an $(n+1)$-bit node channel of a given SPA is configured to receive ATM cells, the protocol is as follows.

1) ATM cells are extracted one word ( $n$ bits) at a time.

2) Cell extraction is initiated when: a) the address within a received fabric header matches the node channel's corresponding SPA fabric address and $b$ ) the destination user is not busy.

3) Extraction ends after the entire ATM cell is received.

4) At all other times, the SPA node channel is transparent, i.e., the optical input data is merely regenerated onto the optical outputs of the node channel.

\section{A. Contention in HyperPlane-Based ATM Switches}

In a HyperPlane-based ATM switch, many of the node channels of a given SPA operate in the receiving mode. This is a direct consequence of using a fixed transmitted assignment. Since each node channel operates independently, it is possible that multiple ATM cells will arrive at the SPA simultaneously on different node channels. Furthermore, the potential exists that two or more of these simultaneously arriving ATM cells will have matching destination addresses. In this case, the destination SPA for these ATM cells will be required to extract multiple cells simultaneously. If the SPA is unable to do this, a collision occurs, at least one ATM cell is lost, and overall switch performance is compromised. One way to overcome this limitation is to add output queuing to the SPA. This enhanced SPA architecture allows ATM cells arriving simultaneously to be temporarily buffered before final delivery to the user. By using this strategy, the fabric cell loss probability is significantly reduced [1], [2].

\section{B. ATM Cell Loss Priority}

While the inclusion of output queuing enhances switching performance considerably, bursty traffic conditions can still lead to queue exhaustion and consequent cell blocks. In these situations, where one cell must be "chosen" over another, it is useful to have an indicator of each cell's relative importance. To that end, the ATM standard incorporates a cell loss priority (CLP) bit [3]. The CLP bit is a part of an ATM cell's 5-byte header, and it identifies the cell as either high priority (CLP $=1$ ) or low priority $(C L P=0)$. For the ATM cells sent on this HyperPlane, the CLP bit is included in the prepended fabric header alongside the destination address of the cell. By including the CLP in the prepended fabric header, extracting SPA node channels are able to determine not only an arriving cell's destination address, but also the cell's priority level. The CLP is then used to determine which ATM cell should be dropped in the event of a contention.

\section{Output Queue Arbitration}

When output queues are added, efficient operation of the SPA becomes dependent upon the effective utilization of those output queues. We earlier defined a protocol where ATM cell extraction is initiated when: 1) a valid fabric header is identified and 2) the destination user is not busy. Obviously, interpreting when a destination user is not busy is less clear when output queues and the CLP bit are added to the switch 
architecture. To that end, a revised methodology for initiating extractions off the HyperPlane is now presented.

The algorithm to initiate extraction when a valid fabric header is received is as follows.

1) Choose an output queue for buffering.

a) If a given queue is empty, extract the incoming ATM cell into the empty queue.

b) If all queues are full and the incoming cell has CLP $=1$, extract the incoming cell into a queue which contains a cell having CLP $=0$.

c) If a suitable queue for extraction is not found from a) or b), the incoming ATM cell cannot be extracted and is lost.

2) Mark the chosen queue as full and note the CLP of the ATM cell being extracted.

3) Order the queue (with respect to the other full queues) for delivery to the user. A FIFO arbitration scheme is used.

The algorithm for delivering ATM cells from the queues to the user is as follows.

1) Select the queue for cell delivery by using the FIFO ordering established during extraction.

2) Deliver the cell from the selected queue to the user.

3) Mark the queue as empty.

\section{A BUFFERED ATM HYPERPLANE SPA}

\section{A. Fabricated SPA Chip}

A SPA which implements the ATM HyperPlane architecture discussed has been designed and fabricated. The system described here uses hybrid-SEED (self-electrooptic effect device) SPA chips [4], in which arrays of differential reflectionmode GaAlAs optical modulators and detectors are integrated with silicon CMOS VLSI, via flip-chip bonding [5], [6] of the optoelectronics directly onto the CMOS circuitry. This allows an efficient pixellated structure to be realized, in which decisions on the incoming optical data can be made local to the optical inputs and outputs, leading to parallel wholeword processing and reduced requirements for driving long capacitive traces on the chip. ${ }^{1}$

A photograph of the chip and a corresponding layout template are shown in Fig. 2. The SPA core is composed of individual smart pixels organized into node channels. The control logic arbitrates the flow of data into and out of the ATM cell queues. Each of the three on-chip queues functions to buffer an entire ATM cell (53 octets of data). The queue addressing blocks are used by the control logic to place each octet of the incoming ATM cell into the queue at the appropriate location. The output multiplexer is used by the control logic to select which of the three queues writes output data to the electrical pinouts of the chip.

The $4 \times 9$ SPA core is comprised of four node channels of nine smart pixels each. Implementing the $4 \times 9$ SPA core

${ }^{1}$ Chip fabrication was provided through the CO-OP sponsored ARPA/AT\&T Hybrid-SEED Workshop held in July 1995 [7]. The workshop provided a $2-\mathrm{mm} \times 2-\mathrm{mm}$ CMOS die with 200 SEED's. The silicon process used had a minimum feature size of $0.8 \mu \mathrm{m}$.

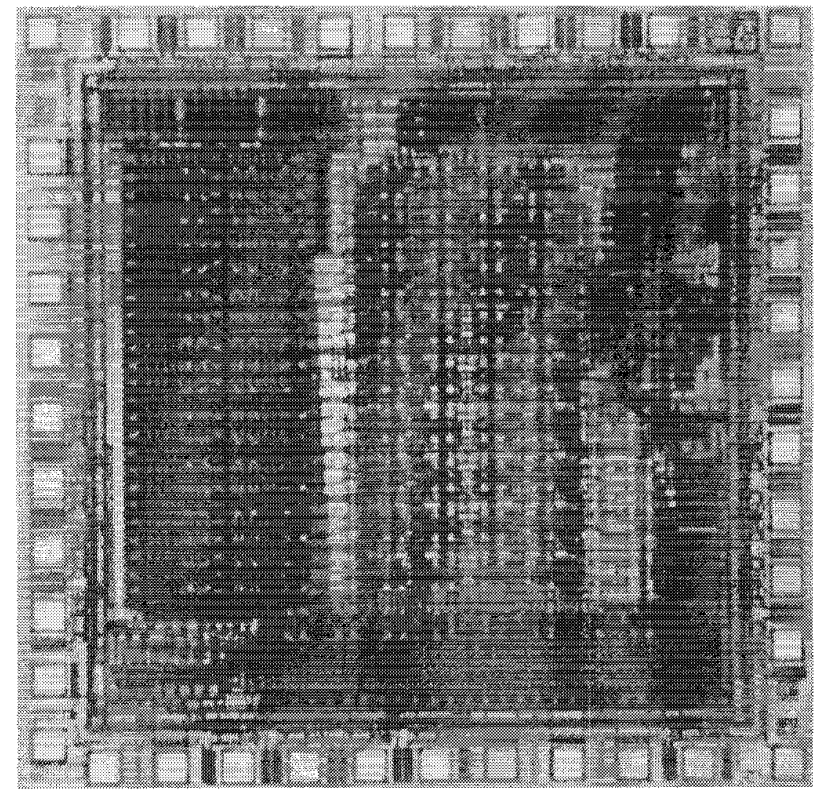

(a)

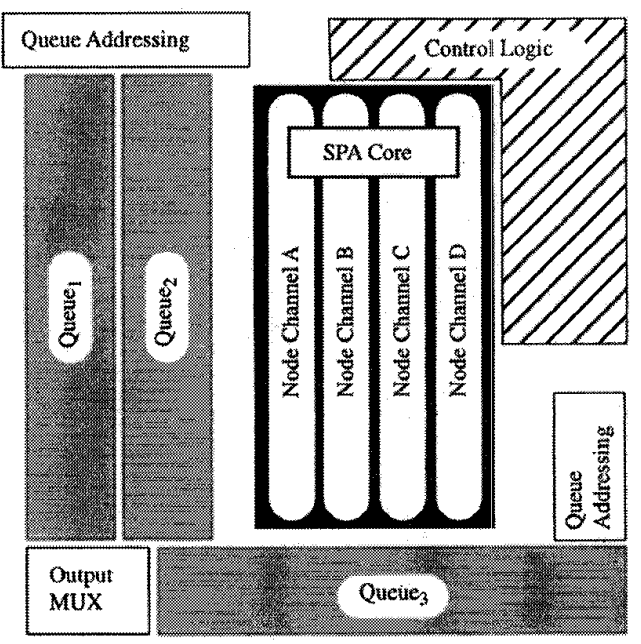

(b)

Fig. 2. SPA photograph and layout template.

used 144 of the 200 SEED's available on the Workshop die. Each smart pixel contains about 60 transistors. The smart pixel circuitry used in this design in shown in Fig. 3. The smart pixel operation is synchronous, so that the optical input data is latched into the pixel by a global system clock. Once latched, the bit can be operated on in several ways depending on the smart pixel state (or, more generally, the node channel state). If the smart pixel is part of a node channel which is configured to received ATM cells, two possible smart pixel states exist. The idle state of smart pixel operation is transparency. During this state of operation, the address recognition circuitry, an XNOR gate, compares the incoming data with the appropriate SPA address bit. The result of this bitwise comparison is used in conjunction with the address recognition results from other smart pixels in the node channel to validate fabric headers and 


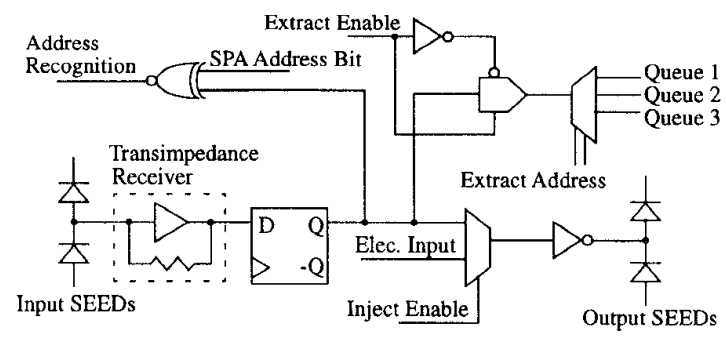

Fig. 3. Smart pixel schematic.

thus initiate ATM cell extraction. In this operating mode, the optical data received is regenerated onto the optical output on the next clock cycle. The second possible state for a "receiving" smart pixel is extraction. During extraction, ATM cell data is extracted off the HyperPlane and into one of the three queues. The control logic sets extract enable HIGH and generates the extract address to designate the appropriate queue for cell extraction. Alternatively, the smart pixel could be part of a node channel which is configured to send ATM cells. In that mode of operation, the smart pixel injects data onto the HyperPlane from the electrical input.

Each queue is implemented as an independent, sequentially addressed, dual-port static random access memory (SRAM). Each SRAM stores 424 bits (53 8-bit rows). The SRAM used is based on an eight-transistor static memory cell which occupies an area of $20.5 \mu \mathrm{m} \times 22.5 \mu \mathrm{m}$. The dual-port design of the SRAM allows for the simultaneous reading and writing of data so that latency in the queuing mechanism is significantly reduced. The simulated read cycle and write cycle times for the SRAM's are 2.5 and $1.0 \mathrm{~ns}$, respectively.

The control logic manages the flow of ATM cells into and out of the three queues. The state machine which determines the current state of the SPA and of the three output queues has 21 latches.

The total number of transistors on the $2-\mathrm{mm} \times 2-\mathrm{mm}$ die is over 20000 . The number of effective transistors per smart pixel is a metric which expresses the complexity of a given SPA. The number of effective transistors per smart pixel is defined as the total number of transistors on the die divided by the total number of smart pixels. For the buffered ATM SPA fabricated, this metric gives $\sim 550$ effective transistors per smart pixel $(=20000$ transistors $\div 36$ smart pixels $)$.

Extensive simulations were performed in the switch level simulator IRSIM to verify the SPA die layout and the correctness of the control logic algorithms. To demonstrate here that these simulations were correct, we present an example of one of the more complex arbitration operations. Fig. 4 shows a particular IRSIM waveform output and provides an example of the control logic's flexibility and functionality in arbitrating the ATM cell queues. Prior to the time frame shown in Fig. 4, high priority ATM cells (CLP $=1$ ) have been extracted into two of the three ATM cell queues. At the beginning of Fig. 4, a lowpriority cell $(C L P=0)$ begins extraction into the third queue. This extraction is shown by the transition on "b.ee" (Node Channel B Extract Enable) and the subsequent increments on the third queue's write addressing lines ("2cnt"). Normally, this ATM cell extraction would continue for 53 clock cycles, as 8 bits are extracted from Node Channel B on each cycle. However, in this instance, the extraction is interrupted by events on Node Channel C. While Node Channel C is initially idle (cin = "000"), a high-priority ATM cell arrives on Node Channel C during Node Channel B's extraction. This highpriority arrival is marked by the ATM cell's fabric header (cin = " $120 ")$. When this fabric header is recognized by the control logic, it looks to find an available queue according to the algorithm presented in Section II-C. In this case, the only "available" queue is one which already contains a low-priority packet. Thus, based on the CLP bit of the two packets, the control logic ends extraction on Node Channel $\mathrm{B}$ and begins a new ATM cell extraction on Node Channel C. This is seen in Fig. 3 when b.ee $\Rightarrow 0$, c.ee $\Rightarrow 1$ (Node Channel C Extract Enable), and when $2 \mathrm{cnt}$ resets and begins incrementing from "00."

In addition to verifying correctness, IRSIM was used to identify the critical path, which was found to limit the system clock frequency to $125 \mathrm{MHz}$. The maximum operating frequency can be used to find an aggregate optical throughput of $4.5 \mathrm{~Gb} / \mathrm{s}(=36$ smart pixels $\times 125 \mathrm{Mb} / \mathrm{s}$ per smart pixel $)$ for the buffered ATM SPA chip which has been fabricated. In addition, the data throughput of each node channel is 1.0 $\mathrm{Gb} / \mathrm{s}(=8$ smart pixels $\times 125 \mathrm{Mb} / \mathrm{s}$ per smart pixel). This node channel throughput is enough to provide each user with a $622-\mathrm{Mb} / \mathrm{s}$ SONET STS-12 link.

\section{B. SPA Chip Extensibility}

The buffered ATM HyperPlane SPA can also be utilized as a building block for larger, more complex SPA's. Used as such, the SPA presented here would be considered as a smart pixel cluster [8]. A 2-D array of these clusters would then comprise the new SPA. The construction of the clustered SPA is accomplished by tiling the buffered ATM HyperPlane SPA (as shown in Fig. 9) and then adding appropriate control logic. Also, another layer of queuing is needed, which changes the queuing characteristics of the SPA from the KnockOut behavior of the individual smart pixel clusters to a CrossOut characteristic across the clustered SPA. This change in queuing structure is accompanied by an increase in HyperPlane switch performance [1], [2].

The SPA chip designed and fabricated to date partitions an aggregate optical system throughput of $4.5 \mathrm{~Gb} / \mathrm{s}$ between four node channels (or users) of nine smart pixels each, all on a single chip. We now address how an ATM HyperPlane switch with $1 \mathrm{~Tb} / \mathrm{s}$ of aggregate optical throughput would be partitioned at the smart pixel, node channel, SPA chip, and PCB levels. We assume that the optics can support 1000 smart pixels per chip and that five SPA chips will be used to support the $1 \mathrm{~Tb} / \mathrm{s}$ of aggregate optical throughput. This is in accordance with the hardware constraints presented in Section $\mathrm{V}$. We begin the analysis by selecting the SONET STS-48 standard for the user interface. This standard provides each user with $2.488 \mathrm{~Gb} / \mathrm{s}$ of bandwidth. The number of users in the system is then $400(=1 \mathrm{~Tb} / \mathrm{s} \div 2.488 \mathrm{~Gb} / \mathrm{s}$ per user $)$. Current shelf-based systems can typically contain 20 PCB's on 1-in spacings, so that 20 users will be served by each PCB. 


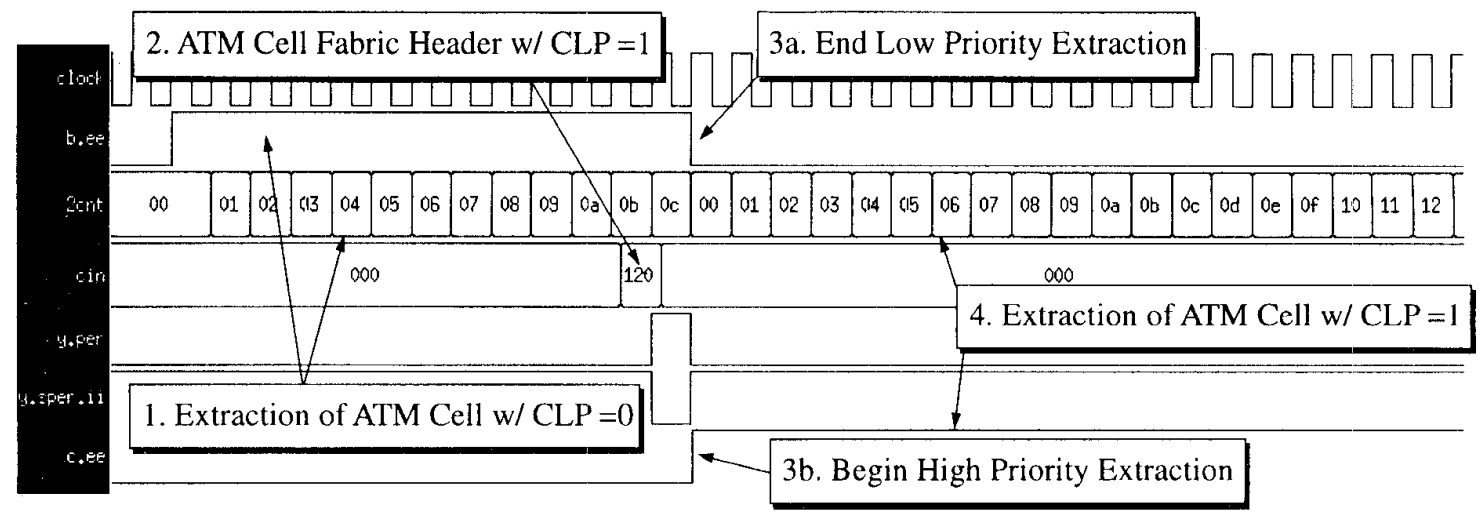

Fig. 4. IRSIM output for queue arbitration based on CLP.

Since a fixed transmitter assignment will be used, 400 node channels are required to support the 400 users. Distributed over five SPA chips, it is found that each chip will support 80 node channels. Each node channel must support the bandwidth of one user $(2.488 \mathrm{~Gb} / \mathrm{s})$, and a tradeoff occurs between the number of smart pixels per node channel and the bit rate of each smart pixel. It is found that a node channel 12 smart pixels wide operating at $207 \mathrm{Mb} / \mathrm{s}$ will provide the needed bandwidth. This gives a total of 960 smart pixels per chip ( $=80$ node channels per chip $\times 12$ smart pixels per node channel). This number of smart pixels per chip is supported by the optics discussed in Section V. In summary, each chip would have $200 \mathrm{~Gb} / \mathrm{s}$ of optical input and $200 \mathrm{~Gb} / \mathrm{s}$ of optical output, and only $10 \mathrm{~Gb} / \mathrm{s}$ of electrical input and $10 \mathrm{~Gb} / \mathrm{s}$ of electrical output, corresponding to 100 electrical $\mathrm{I} / \mathrm{O}$ pins at $200 \mathrm{Mb} / \mathrm{s}$

\section{Optoelectronic InTERface CirCuitry}

\section{A. Receiver}

The optical power reaching each detector is expected to be around $-45 \mathrm{dBm}(30 \mu \mathrm{W})$ high state, and $-48 \mathrm{dBm}(15 \mu \mathrm{W})$ low state, giving a differential optical power of $\pm 15 \mu \mathrm{W}$. The estimation of these incident detector powers is based upon assumptions concerning the interconnect optics and the physical performance of the SEED's. These assumptions are: 1) $10 \mathrm{~dB}$ of laser to receiver optics loss and 2) SEED modulator reflectivities of $60 \%$ (high state) and 30\% (low state). Estimates of the efficiency of all elements in the optical design presented below suggest that the $10-\mathrm{dB}$ loss value is achievable given state-of-the-art diffractive optics fabricated by optical lithography. It is important to maintain this optical loss target, as higher optical losses require the use of either more complex, higher power receivers or increased laser source power for a given bit-error rate, both of which are undesirable.

Transimpedance receivers have been used previously in hybrid-SEED chips because of their excellent bandwidth and dynamic range performance [9]. The transimpedance receiver stage used in our SPA chip is shown in Fig. 5. The receiver was designed to operate at low signal levels with minimal signal propagation delay. SPICE simulations indicate the prop-

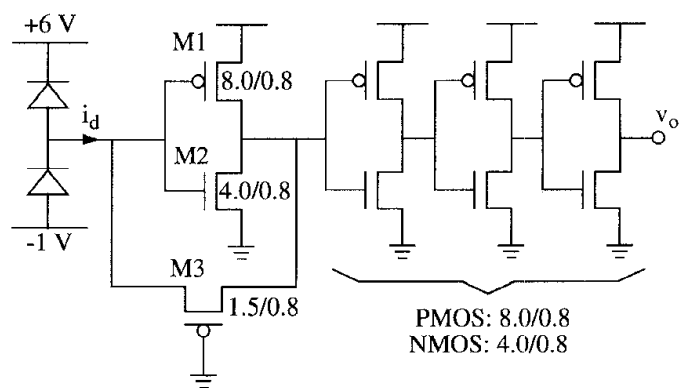

Fig. 5. Transimpedance receiver schematic (W/L in $\mu \mathrm{m})$.

agation delay through the receiver to be less than $2.0 \mathrm{~ns}$ with $\pm 10 \mu \mathrm{W}$ of differential optical power at the input SEED's. The receiver is estimated to dissipate $7.5 \mathrm{~mW}$ when operating with $\pm 10 \mu \mathrm{W}$ received differential power at $125 \mathrm{Mb} / \mathrm{s}$. Additional SPICE characterization of the transimpedance stage (M1, M2, M3) found the transimpedance gain $\left(Z_{T}\right)$ to be $84 \mathrm{~dB}$, the bandwidth $\left(f_{3-\mathrm{dB}}\right)$ to be $410 \mathrm{MHz}$, and the transimpedancebandwidth product (TZBW) to be $69 \mathrm{GHz} \cdot \Omega$. The receiver occupies a total chip area of $35 \mu \mathrm{m} \times 40 \mu \mathrm{m}$.

\section{B. Transmitter}

The optical power incident on each modulator SEED window in our chip is chosen to be $160 \mu \mathrm{W}$. This would correspond to $1 \mathrm{~W}$ of source laser power per chip and 5-dB laser to modulator optical loss in the case of a 1000-smartpixel chip. The electronic circuitry to drive these modulators consists of a single CMOS inverter (PMOS: $32.0 / 0.8 \mu \mathrm{m}$, NMOS: $16.0 / 0.8 \mu \mathrm{m}$ ) driving a differential SEED pair (biased at $+6 /-1 \mathrm{~V}$ ). This single-stage output is sufficient to modulate the optical output in excess of $1 \mathrm{GHz}$, taking into account both capacitive loads and photocurrent loads.

Aside from the simple issue of laser availability, it is desirable to keep the laser power low since the incident power at the optical modulators generates electrical heating through the photocurrent. Assuming 0.3-A/W average SEED sensitivity and 6-V SEED bias, the total heat load due to photocurrent in the modulators would already be around 0.6 $\mathrm{W}$ for a total laser power of $1 \mathrm{~W}$ per 1000 -smart-pixel chip, which would be a significant fraction of the total chip heat load. 


\section{OpTICS}

\section{A. Hardware Constraints}

There are a number of hardware limitations which drive us to the particular physical division of the total $1-\mathrm{Tb} / \mathrm{s}$ bandwidth described in Section III-B. The optics described below only support unidirectional operation, and optically closing the ends of the backplane to form a ring would be very difficult. Hence, separate chips for up- and down-node channels are required. Clock speeds of present-day high-performance commercial CMOS are around $100-300 \mathrm{MHz}$ (in particular, the chip described above has a clock speed of $125 \mathrm{MHz}$ ), so that around 5000 parallel channels in each direction would be needed for $1-\mathrm{Tb} / \mathrm{s}$ bisection bandwidth. The volumetric overhead of both the electrical packaging and of the optomechanics suggest that the best way to implement the $1 \mathrm{~Tb} / \mathrm{s}$ is to use the smallest possible number of the largest available chips. Therefore, the optical channels could most conveniently be divided between 10 identical chips per PCB (five in each direction), with each chip containing 1000 smart pixels. Since each smart pixel has a differential optical input and a differential optical output, this requires an optical connection density of 4000 per chip (and hence 4000 SEED's per chip).

Currently, mass-produced CMOS chips are available around $1 \mathrm{~cm}^{2}$ in size. These chips need to be individually packaged, since multichip module technology (MCM) is not applicable to this optical backplane as successive chips on an MCM substrate are not well aligned. Such a $1-\mathrm{cm}^{2}$ chip of 1000 smart pixels would require around 300 electrical pinouts, of which 50 are signals in and 50 are signals out at $200 \mathrm{Mb} / \mathrm{s} / \mathrm{pin}$. Packages supporting this level of pinouts would be on the order of 1 in on a side, given near-future pin grid array or ball grid array technology. A PCB in a switching system frame can reasonably have an edge (or "beachfront") of only around 10 in, which would indeed fit with the required 10 chips per PCB. Hence, we see that the optomechanics must support 1000 smart pixels per chip and must have a 1-in footprint.

Optically interconnecting the SPAs on successive boards requires a repeated optical module which can deliver optical power to the modulators and can then transfer the modulated beams to the detectors on the next board. The HyperPlane backplane architecture outlined above in Section II requires only a simple one-to-one interconnection, thus avoiding the higher optical losses and more complex alignment issues associated with more complex interconnects (shuffles, crossovers, etc.) [10]-[12]. Although such interconnects may offer greater functionality, they have proved difficult to construct in practice [10]. For near-term work, we therefore choose the simplest solution. Further, in our system, although data can propagate board-by-board along the entire backplane, the optical beams are absorbed at each stage so that each optical interconnection is only from one board to the next. This regeneration drastically lowers the optical loss and optical aberration requirements of the module, as compared to possible "transparent" backplane implementations [13]. Clearly, regeneration introduces extra path-dependent latency. However, this is unimportant since ATM cells are not synchronized to a global clock.
The following set of characteristics for the optical module associated with each chip is therefore imposed.

1) Support a channel density of 1000 smart pixels $/ \mathrm{cm}^{2}$.

2) Fit in a standard electrical rack: $\sim 1$-in board spacing, with 1 -in $\times 1$-in $\times 2$-in optomechanics volume per chip.

3) Operate with a $1-\mathrm{cm} \times 1-\mathrm{cm}$ chip size.

4) Keep the loss between the optical power supply and the detector to less than $10 \mathrm{~dB}$.

5) Use only elements which are potentially cost-effective in mass-production.

6) Minimize the number of critical alignments.

A number of optical systems have been built or proposed which address a number of the targets above [10], [14]-[18], but none of them satisfactorily solve the issue of extensibility to $1-\mathrm{cm}$ chip size with low-cost lenses. In particular, SEED-based systems which form the interconnect using only microlenses [14], [15], [19] suffer from the limited throw available from a given size Gaussian beam. In this discussion, we define a microlens as a lens which is small (of the order of $100-\mu \mathrm{m}$ diameter) and supports only one or a few beams. If the chips to be interconnected by a purely microlens system are placed at realistically large separations, then the microlens diameters need to be so large that the number of smart pixels on a 1 -cm-size chip is unacceptably low.

We describe in this section the design of an optical module for a free-space optical backplane which meets the performance criteria listed above by mixing microlenses and minilenses and by using a novel beam combination technique.

To achieve the necessary performance at reasonable cost, we do not use any lenses with a field of view equal to the size of the entire $1-\mathrm{cm}^{2}$ chip. Instead, the chip is divided into clusters, following the work of Rolston et al. [8], with independent optics for each cluster (apart from the common first stage of the optical power supply, and the polarizing elements which have no optical power). The $f / \#$ requirements are thus relaxed by breaking down the large chip into a set of smaller fields. Each field is supported by a transmitter minilens and a receiver minilens, as will be seen below. A chip has been represented in Figs. 6 and 9 as having two clusters in $x$ and four clusters in $y$, and in fact this is probably about the optimum number for a 1-cm-sized chip.

The $f / \#$ of the minilenses in our system is increased further to a reasonable number (around $f / 4$ for the transmitter lenses) by the use of short focal length microlenses to achieve final focusing onto or collimating from the small SEED windows.

The problem of the short throw of Gaussian beams with small waists is addressed to an extent in our system by the demagnification of the microlenses. However, due to the long optical path (which is equivalent to $43.2 \mathrm{~mm}$ of air to interconnect chips which are only physically separated by $25.4 \mathrm{~mm}$ ), the beam throw still limits the connection density, as will be seen from the smart pixel density calculation in Table I. It is clear that the shorter the minilens focal length, the greater will be the smart pixel density for given $f / \#$ 's. Fortunately, this matches the requirement for a compact system in order to fit the available space. Ideally, the optical path would be straight from one chip to the next with the chips 


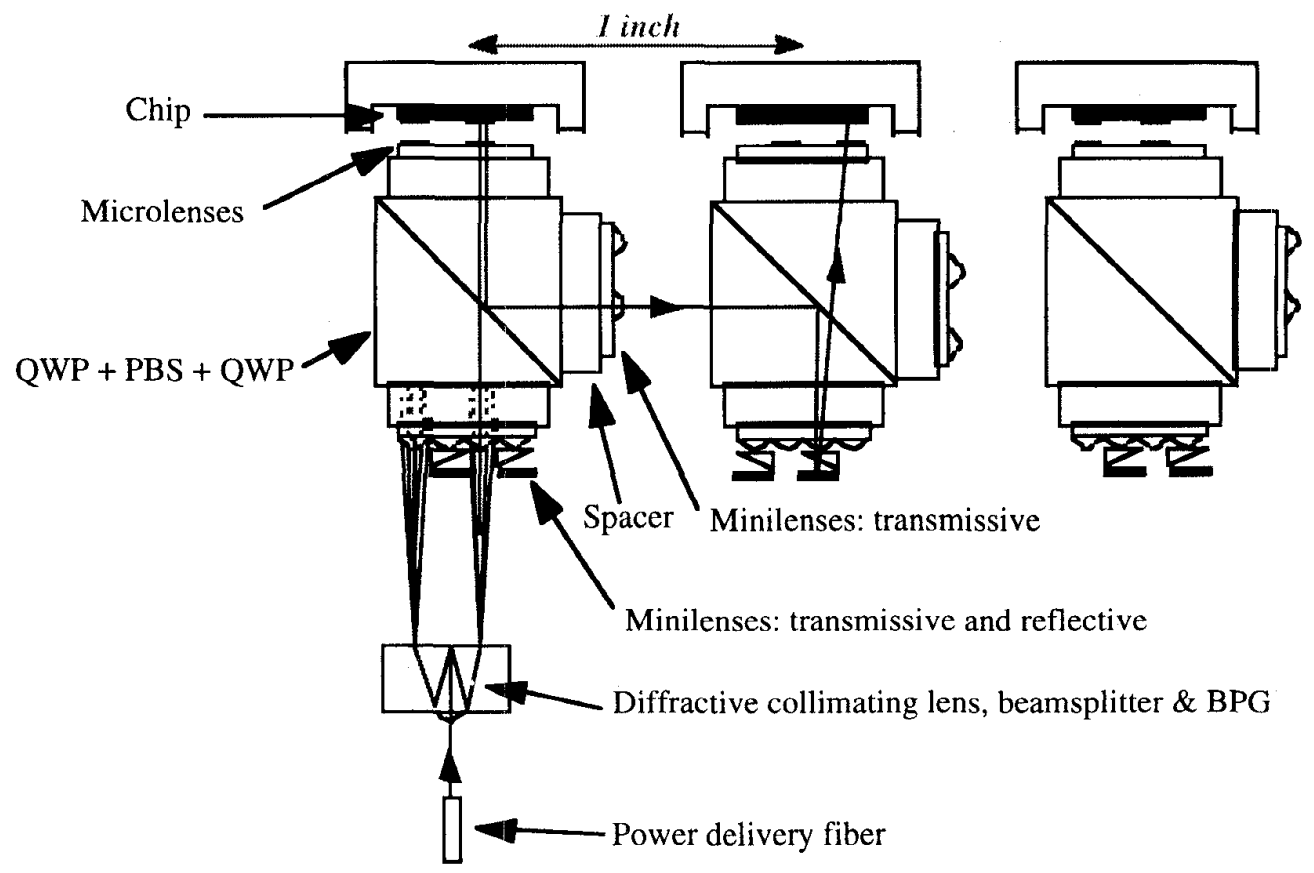

Fig. 6. Compact off-axis receiver optics. Sketch is approximately to scale, with smart pixel chips lying on a 1-in basis. The $x$ axis runs rightwards, $y$ is into the plane of the drawing, and $z$ runs upwards. Power supply is shown for one chip only. Electrical PCB's occupy $y-z$ planes. Modules are repeated to the left and right.

TABLE I

Calculated Parameters of Optical System

\begin{tabular}{l|c||l|c}
\hline Air gap between BPG and minilens & $10.459 \mathrm{~mm}$ & Minilens focal length & $10.459 \mathrm{~mm}$ \\
\hline Microlens/minilens quartz substrate thickness & $0.400 \mathrm{~mm}$ & Minilens A & $-4.773 \times 10^{-8} / \mu \mathrm{m}^{2}$ \\
\hline Polarizing beamsplitter thickness, BK7 & $9.813 \mathrm{~mm}$ & Minilens B & $1.318 \times 10^{-17} / \mu \mathrm{m}^{4}$ \\
\hline Air gap thickness between microlens and SEEDs & $0.350 \mathrm{~mm}$ & Minilens C & $-5.913 \times 10^{-26} / \mu \mathrm{m}^{6}$ \\
\hline Spacer thickness, SF10 & $2.482 \mathrm{~mm}$ & Minilens D & $0.210 / \mathrm{mm}$ \\
\hline Air gap thickness between modules & $12.705 \mathrm{~mm}$ & Microlens focal length & $0.350 \mathrm{~mm}$ \\
\hline Angle of receiver beams in air & $10.12^{\circ}$ & Microlens A & $-1.429 \times 10^{-6} / \mu \mathrm{m}^{2}$ \\
\hline Beam $\omega$ at SEEDs & $5.45 \mu \mathrm{m}$ & Microlens B & $2.914 \times 10^{-12} / \mu \mathrm{m}^{4}$ \\
\hline Beam $\omega$ at microlenses & $18.2 \mu \mathrm{m}$ & Microlens C & $-1.144 \times 10^{-17} / \mu \mathrm{m}^{6}$ \\
\hline Beam $\omega$ at waist in front of microlenses & $17.4 \mu \mathrm{m}$ & BPG compression ratio & 2.25 \\
\hline Beam $\omega$ at minilenses & $163.9 \mu \mathrm{m}$ & Clipping ratio at SEEDs & 3.3 \\
\hline Beam $\omega$ at BPG & $163.0 \mu \mathrm{m}$ & Clipping ratio at \\
& & minilens (corner spot) & 5.8 \\
\hline Connection density & $\mathbf{2 0 6 0 / \mathbf { c m } ^ { 2 }}$ & Smart pixel density & $\mathbf{5 1 5 / \mathbf { c m } ^ { 2 }}$ \\
\hline
\end{tabular}

parallel to the circuit boards. Such a system would have an interconnect length of about $25 \mathrm{~mm}$ of glass, equivalent to just $17 \mathrm{~mm}$ of air, resulting in connection densities which are higher by an order of magnitude than those described here. It will also lead to easier beam combination for a modulator system, or much simpler optomechanics for a system that uses vertical-cavity surface-emitting lasers (VCSEL's) [17], [20]-[22] which require no optical power supply. However, to build such a parallel-chip system will require the development of transparent heatsinks and further work on through-substrate smart pixels [23], [24] in which the receivers and transmitters can in principle operate through opposite faces of the chip.

All the lenses in the system we have designed are planar diffractive elements defined by optical lithography, which should be more cost-effective in mass production than bulk glass lenses since they are made using technology closely related to that of the semiconductor chip industry. They are also highly reproducible and controllable in terms of their focal length and aberration performance. It was found in the system here that they can offer negligible aberration, which helps to reduce optical loss. At present, refractive microlenses such as photoresist bump lenses [25], are not manufacturable to sufficient focal length tolerance to be useful in this application, although refractive lenses produced by mass-transport on $\mathrm{GaP}$ [26] show promise.

The number of critical alignments is minimized in our system by choosing an optical system as close as possible to a telecentric single $4 f$ system. Beam combination here 
is a minilens array adaptation of the pupil-division approach of McCormick et al. [10], with the extra difference that the receiver minilens has an additional prismatic effect. Hence, high optical power efficiency is maintained without the need for image-plane pixellated mirrors. It will be seen that there are still patterned mirrors in the system, but, since they are in the collimated-beam region, their dimensions are of the order of millimeters with alignment tolerances of many tens of microns, and they do not introduce additional low $\mathrm{f} / \mathrm{\#}$ imaging optics. This is an advantage over systems in which the patterned mirrors are at an intermediate image plane, with feature sizes of the same order as the actual modulators [14].

\section{B. Off-Axis Receiver Optical Interconnect}

Fig. 6 shows a new optical layout which we term the "offaxis receiver optics" whose performance meets the system targets and addresses many of the problems with previous work in this field. The figure is drawn roughly to scale, with the chips on a 1-in basis. In Fig. 6, the minilenses have been drawn as refractive lenses and prisms for clarity, but the minilenses and the microlenses are intended to be diffractive. A patterned mirror has also been drawn as a discrete item. It is intended that this is actually a reflective coating on the etched surface of the diffractive minilens/prism element. The purpose of the spacer in this optical layout is to equalize the optical path lengths by compensating for the path length of the quarter wave plate.

Each optical stage is essentially a single $4 f$ system consisting of two minilenses, with microlenses to add focusing power next to the SEED's. The system is therefore well described by focal Gaussian beam analysis once the aberrations are taken into account. Beam waists are located at the fiber facet, at the BPG $\left(1 / e^{2}\right.$ intensity radius $\left.\omega=163 \mu \mathrm{m}\right)$, and at one microlens focal length before $(\omega=17.4 \mu \mathrm{m})$ and after each microlens $(\omega=5.45 \mu \mathrm{m})$. This last waist is at a SEED window.

The SEED windows are clustered in small arrays, and a clustered microlens array is positioned above the chip, such that one transmitter window lies above the center of each microlens, and a receiver window lies next to the transmitter window, but off-axis with respect to the microlens. A close-up of this is shown in Fig. 7. The microlens dimension is equal to the SEED transmitter spacing (the microlenses are assumed to have no margins around them, although in practice only the central portion of each microlens is used by the optical beam). Obviously, implementing a smart pixel consisting of a differential SEED transmitter and a differential SEED receiver requires exactly two microlenses.

To interconnect a set of SEED chips, beams propagating in three directions in the same space are required. A polarizing beamsplitter/quarter-wave plate element can only overlay two of these sets of beams. A number of different methods to add in the third set have been demonstrated (for a review, see [27]), but none of the methods demonstrated to date simultaneously satisfies the requirements of low optical loss, single-wavelength operation, and applicability to the case of a $1-\mathrm{cm} \times 1-\mathrm{cm}$ chip without using a single expensive lens for the whole chip. In our system, the minilenses on the receiving arm have additional prismatic power, achieving beam combination

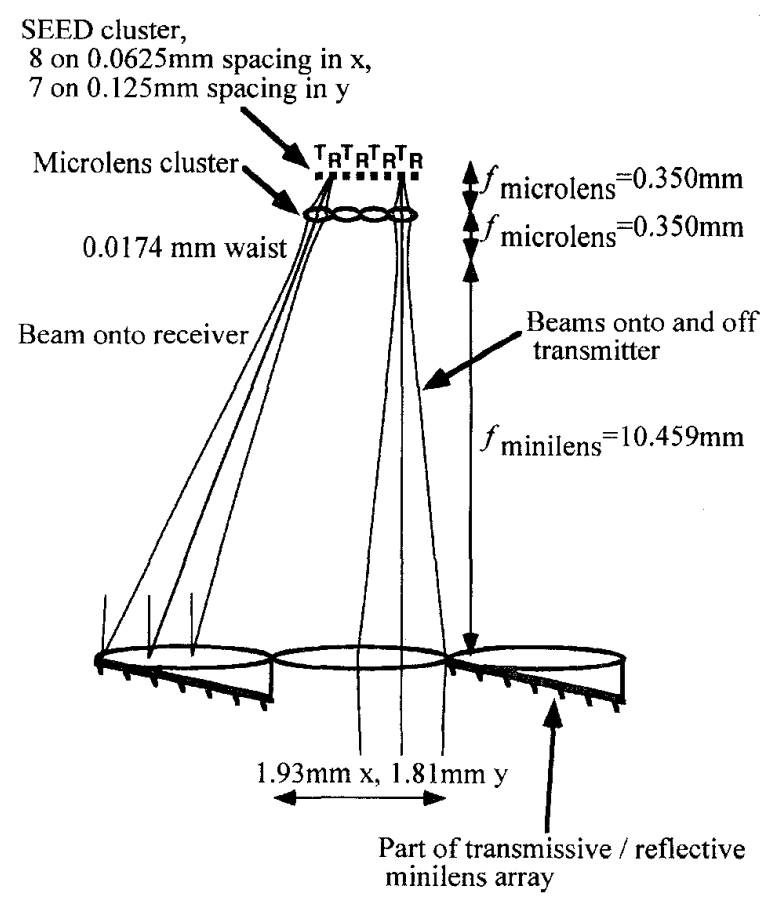

Fig. 7. Close-up of off-axis receiver optics, showing two representative beams. Each SEED pair has a transmitter SEED on-axis to the respective microlens and a receiver SEED off-axis.

by interlacing the beams for the receivers at the chip plane with those for the transmitters (see Fig. 7).

It may be seen that successive optical modules have minilens and mirror arrays which are rotated by $180^{\circ}$. When the beam modulated by one smart pixel chip reaches the transmissive/reflective minilens array on the next module, it hits a reflective receiver minilens, which includes a prism implemented as a digitized, modulo $2 \pi$, linear phase gradation, superimposed on the diffractive minilens. The light therefore leaves the minilens at a precalculated angle, such that it hits the center of the corresponding microlens. The receiver minilens has the same focal length as the transmitter minilens and so, due to the angle of the new beam, the system is no longer exactly focal. It is found, however, that this has a negligible effect on the spot size at the receiver SEED. The chief ray of the receiver beam is undeviated at the microlens since it passes through the center of the microlens, and therefore the receiver SEED window is off-axis with respect to the microlenses. Hence, the modulated light now forms a waist on a receiver SEED window, where it is detected and amplified by the receiver circuit.

A problem with this system is the lack of telecentricity on the receiving arm at the microlenses and at the receiver windows, resulting in tight tolerances on the receiver minilens focal length, the prism angle, the microlens focal length, and the separation between the minilens and the microlens, and between the microlens and the chip. Since the first three of these are lithographically defined, only the latter two present any difficulties. Careful optomechanical assembly techniques will be required to overcome these issues. 
Aberrations or misalignments of a given size have a bigger effect on the overall optical power efficiency from laser to receiver and on the optical crosstalk when they occur early in the optical path than they do when they occur later in the path. The beams in our system are constrained to be either onaxis or at least telecentric for all the optics up to the last two lenses. Hence, the major aberrations and tightest tolerances have been reserved for the final sections of the optical path, where they will have the least effect on received power and receiver crosstalk.

\section{Optical Power Supply}

For stability, power supply lasers in SEED systems need to be either grating-tuned or distributed-feedback/distributedBragg-reflector structures [10], [28], which are very expensive. It is therefore desirable to use only a single high-power laser for each chip. In addition, it is especially important to consider the optical power supply in this system, since we wish to cause light from a single source to reach the whole of the 1cm-sized chip, but without incorporating any expensive $1-\mathrm{cm}$ field-of-view lenses.

The optical power supply generates the original clusters of beams to read the modulators. The layout of one of these clusters as needed to match the small test smart pixel chip described in Section III is shown in Fig. 8, and the manner in which these clusters would be tiled to power a large chip is represented in Fig. 9. The optical power supply is shown for only one chip in Fig. 6, but clearly one of these units would be required for every chip. It has a cascaded design, in which initially one beam is generated for each cluster; this beam in turn generates the array of spots for its own cluster using a binary phase grating (BPG) which is positioned at the front focus of the corresponding Fourier transform minilens. The result is a widely-spaced set of closely-spaced clusters of spots. All the spots in Fig. 8 are from one transmissive minilens and each spot hits one of the transmitter SEED's in a certain cluster. The optical axis shown is for the first minilens. The slight asymmetry is due to the choice of an even-orders missing [29] BPG design generating $10 \times 8$ spots, of which one column is thrown away, matching the $9 \times 8$ set of transmitter SEED's. It may be seen in Fig. 9 that the need for a receiver minilens requires extra space between the smart pixel clusters. However, this can be used very effectively to allow implementation of the powerful two-layer queuing system described in Section III-B above.

The required clustered beams could in principle all be generated using a single conventional free-space transmissive BPG. However, since the full-field diagonal separation of the outermost of these beams is roughly $12 \mathrm{~mm}$, a long focal length $(f \sim 90 \mathrm{~mm})$ Fourier lens would be needed to maintain a realistic $f / \#$. To meet the system volume requirement, the optical path would then need to employ discrete elements in a folded configuration. This would be complex.

The three components of the power supply optics can potentially be lithographic and combined onto one double-sided substrate as drawn in Fig. 6, resulting in lower fabrication costs, simplified mechanics, and reduced system volume when

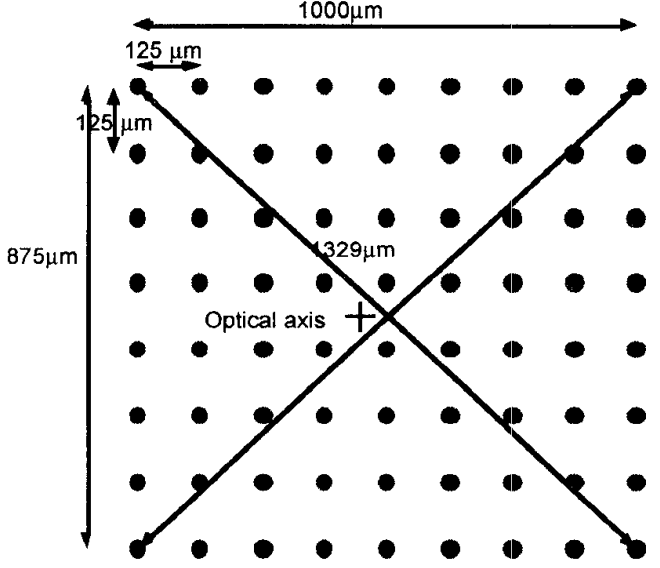

Fig. 8. Spots from optical power supply for one cluster. Spot sizes shown are not to scale.

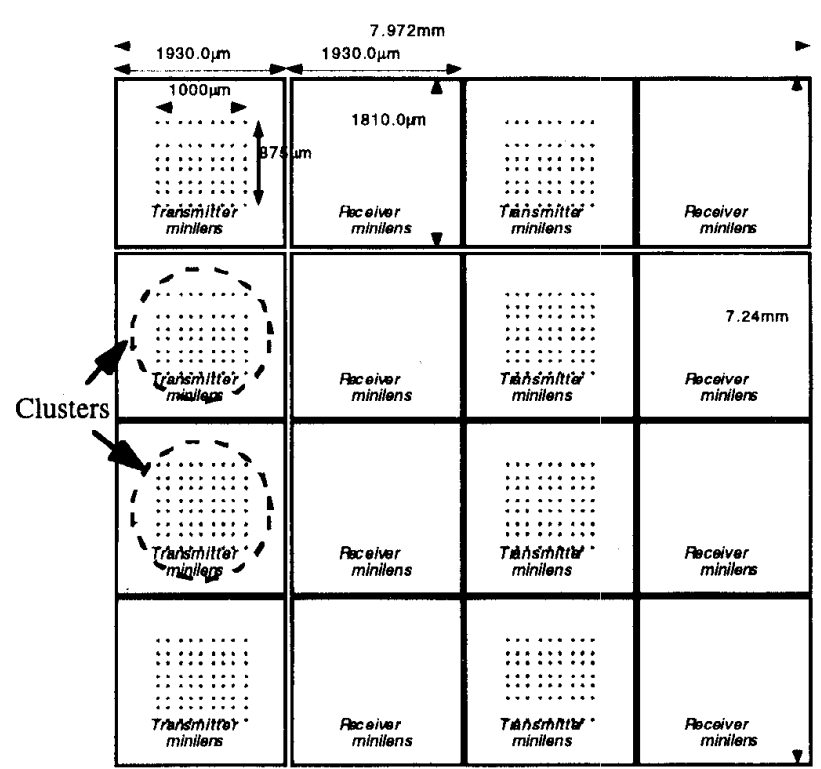

Fig. 9. Typical set of minilenses and clusters of spots needed from optical power supply for a large chip, based on the chip of Fig. 8 as repeat unit. A smart pixel cluster lies under each transmitter minilens and is supported by that minilens and the neighboring receiver minilens.

compared to conventional discrete-element systems [15]. An integrated minilens collimates the light from a remote laser which is brought in by a single-mode polarization-preserving fiber (as shown), or from the local power supply laser diode. The first-stage beams are then generated using a diffractive beamsplitter with multiple bounces inside a thick substrate [30]. This generates a set of large $(\omega=163 \mu \mathrm{m})$ widelyspaced, parallel, collimated beams. Beam diffraction due to the multiple-bounce path length through the substrate is usually a limiting factor for integrated beamsplitters, but in this case the Rayleigh range of the beam in glass is about $15 \mathrm{~cm}$. Therefore, the path length within an integrated beam splitter between the first reflection and the exit from the substrate for the most offaxis spot (6 $\mathrm{mm}$ from the axis) would be only $1 / 3$ Rayleigh 
range for realizable internal diffraction angles of $7^{\circ}$. Hence, the integrated beamsplitter should be a feasible solution here.

\section{Optical System Design Process}

The transmissive diffractive lenses were expressed as a polynomial phase expression of the form

$$
\phi(r)=\frac{2 \pi}{\lambda}\left(A r^{2}+B r^{4}+C r^{6}\right)
$$

where $\phi(r)$ is the phase shift of the wavefront on passing through the lens as a function of radial distance $r$ from the center of the lens, $\lambda$ is the wavelength in air, and $A, B$, and $C$ are constants to be found for each type of lens. Once the constants were found, the phase expression was divided by modulo $2 \pi$ and digitized into four or eight phase levels. Arrays of lenses with this phase behavior were then laid out in the GDS-II mask-making format. The reflective minilenses were modeled as a combination of two transmissive lenses in succession, with a zero-thickness gap between them (the turning induced by the reflection is not relevant to the optical modeling). They are similar to the transmissive minilenses, except that they have exactly half the focusing power on a single pass (and hence the same focusing power given two passes) and have an additional linear phase gradation. Hence, the phase behavior of these lenses may be expressed as

$$
\phi_{\text {reflective }}(r)=\frac{2 \pi}{\lambda}\left(\frac{A}{2} r^{2}+\frac{B}{2} r^{4}+\frac{C}{2} r^{6}+\frac{D}{2} x\right)
$$

where $\phi_{\text {reflective }}(r)$ is the phase shift of the wavefront on each pass of the reflective lens, $A, B$, and $C$ are the phase constants of the transmissive minilenses, $D$ is the phase constant needed to produce the angular deviation, and $x$ is the lateral distance across the lens. The etch depth of the reflective minilenses is shallower for a given phase shift than for the transmissive lenses since the reflecting medium is to be coated directly on the etched surface without an air-gap.

The microlens and minilens phase constants were determined by automatic optimization using the OSLO optical design package. ${ }^{2}$ The optimization target was for the chief ray of all the beams in one cluster to follow the correct path from the BPG to the receiver SEED's and also to minimize the geometric ray-traced spot size at the required beam waist positions for the on-axis and most off-axis spots in the case of the transmissive minilens, and the read-out beam on a given SEED in the case of the microlens. The test ray set for a given spot was Gaussian apodized with the beam size calculated by Gaussian beam analysis. It was found that with just three polynomial constants in the phase expressions, the geometric spot sizes predicted were orders of magnitude lower than the Gaussian beam (diffractive) spot sizes, with Strehl ratios typically a remarkable 0.9999 , and the chief rays of all the beams in the cluster were within $0.4 \mu \mathrm{m}$ of their target positions at all points from the BPG to the receiver minilens, even including the effect of $\sin (\theta)$ distortion by the BPG. Hence, the system as modeled is effectively diffraction limited and distortion-free.

\footnotetext{
${ }^{2}$ Sinclair Optics, Inc., Fairport, NY.
}

\section{E. Constraints and Performance of Optical Design}

Three constraints have been imposed in the optical design. The first constraint is that the compression ratio $R$ of the spot array generator must be sufficiently large [27], where

$$
R=\frac{\text { separation of odd order spots }}{\text { spot size }}
$$

with both parameters measured in the Fourier plane. The relevant spot size here is $3 \omega_{\text {waist }}$, where $\omega_{\text {waist }}$ is the spot radius at the waist just in front of the microlens. Hence, for this system

$$
R=\frac{D_{\text {microlens }}}{3 \omega_{\text {waist }}}
$$

since the odd-odd spot separation is equal to the microlens

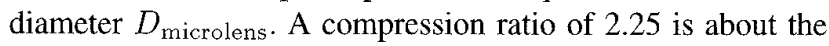
minimum permissible value for good power uniformity across the array [27], [31], although this value depends on the details of the particular BPG design and fabrication.

The second constraint concerns the $f / \#$ of the diffractive lenses. A typical eight-level (three mask steps) diffractive lens process using optical lithography can only achieve $f / 8$ at present before the diffraction efficiency begins to fall off at the edge of the lens. The edge of the lens is where the phase gradient is steepest and therefore the digitized steps most closely approach the fabrication process resolution. Therefore, it is desirable to keep the lens diameters small for a given focal length. However, this results in clipping of the beams at these elements. This results in a power loss and in a change in the parameters of the Gaussian beam after the clipping element. The latter effect dominates for the clipping ratios of interest here [32]. Clipping may be neglected if the clipping ratio $C$ is at least 4.25 , where $C=D_{\text {element }} / \omega_{\text {beam }}$, where $D_{\text {element }}$ is the diameter of the element and $\omega_{\text {beam }}$ is the beam radius at that element. In the analysis here, a square lens or window is approximated as a circle of diameter equal to the side of that square; the difference in practice is about a factor of two in the clipped power, but to fully analyze the system effect of clipping a round beam by a square aperture would be exceptionally difficult.

The aperture stop of this system is at the BPG, so as to make the system telecentric up to just before the receiver minilens. Therefore, a beam not at the edge of a cluster will be well away from the edges of the minilenses and essentially will not be clipped at all by the minilenses. It is the clipping of the corner beams of each cluster which was therefore considered in determining the size of the minilenses.

The final constraint is that the effective SEED window diameter is $18 \mu \mathrm{m}$ [7], and the SEED transmitter windows are on a $125-\mu \mathrm{m}$ grid in $x$ and $y$, with a receiver window lying $62.5 \mu \mathrm{m}$ along $x$ from each transmitter window. This is not the optimal SEED positioning but is a constraint of the given hybrid-SEED design rules. The SEED size corresponds to a $20-\mu \mathrm{m} \times 20-\mu \mathrm{m}$ device with a $1-\mu \mathrm{m}$ implanted margin on each side [7], which was approximated in the modeling by a circle of $18-\mu \mathrm{m}$ diameter.

The optical data calculated for the system is shown in Table I. The layout of the windows and the dimensions of the 
minilenses are shown in Figs. 8 and 9. It is seen that all the constraints are met, with the exception that the beamsplitter is marginally too small to allow a full $1-\mathrm{cm} \times 1-\mathrm{cm}$ chip. A smart pixel density of $515 / \mathrm{cm}^{2}$ averaged over the entire minilens array is achieved. This is within a factor of two of the target of 1000 smart pixels $/ \mathrm{cm}^{2}$. Further analysis showed that a smart pixel density of $1125 / \mathrm{cm}^{2}$ could be achieved with different SEED positioning, using a different set of lens parameters. In this higher density case, each cluster contains nine transmitter windows along $x$ and 16 along $y$ on a $84-\mu \mathrm{m}$ square grid, and the receiver windows are displaced from the transmitters by $33 \mu \mathrm{m}$ along $x$.

The microlenses are used by each Gaussian beam at $f / 6.4$, where the beam diameter is taken as $3 \omega$. Similarly, the minilenses are used by each beam at $f / 21.4$. The transmitter minilens as a whole is $f / 3.9$. The receiver minilens is about $f / 4.4$ on each pass. Given the optical lithography diffractive optic process we are using, the microlenses can be made with an eight-level process across the entire region which the beam hits, while the minilenses require a four-level process toward the edges. This results in a slightly decreased diffraction efficiency for the outermost beams at the minilenses.

About half of the apparent power of the receiver minilenses comes from the prismatic effect needed for the angular deviation; the actual focusing power of these lenses on a single pass is exactly half that of the transmitter minilenses. A refractive miniprism could be integrated with the receiver minilens, using for instance ion exchange or surface-profiling [33]. The diffractive part of this element would then be an $f / 8.8$ lens, which could be built with eight phase levels. Incorporating this refractive miniprism would, however, greatly increase the fabrication complexity.

\section{CONCLUSION}

The implementation of an optical HyperPlane-based ATM switching fabric has been presented. Key hardware components for this system, namely the SPA circuitry, the optoelectronic interface, and the optical interconnect, have been designed and are currently being fabricated using technologies which are available today. This has allowed us to conclude that the goal of an intelligent backplane with $1-\mathrm{Tb} / \mathrm{s}$ bisection bandwidth is realizable using free-space optical connections, and that such a system will offer significant performance advantages over all-electronic backplane implementations.

\section{ACKNOWLEDGMENT}

The authors wish to thank W. M. Robertson for designing the BPG and A. Fedor for coding the diffractive optics masks. The smart pixel chips and most of the diffractive optics have been fabricated by AT\&T and Honeywell, respectively, through the ARPA CO-OP.

\section{REFERENCES}

[1] T. H. Szymanski and H. S. Hinton, "Design of a terabit free-space photonic backplane for parallel computing," in Proc. 2nd Int. Conf. Massively Parallel Processing Using Optical Interconnections, San Antonio, TX, Oct. 1995, pp. 16-27.
[2] H. S. Hinton and T. H. Szymanski, "Intelligent optical backplanes," in Proc. 2nd Int. Conf. Massively Parallel Processing Using Optical Interconnections, San Antonio, TX, Oct. 1995, pp. 133-143.

[3] ATM User Network Interface Specification, v. 3.1

[4] A. L. Lentine, K. W. Goossen, J. A. Walker, L. M. F. Chirovsky, L. A D'Asaro, S. P. Hui, B. Tseng, R. E. Leibenguth, D. P. Kossives, D. W. Dahringer, D. D. Bacon, T. K. Woodward, and D. A. B. Miller, "Arrays of optoelectronic switching nodes comprised of flip-chip-bonded MQW modulators and detectors on silicon CMOS circuitry," IEEE Photon. Technol. Lett., vol. 8, pp. 221-223, 1996.

[5] M. J. Goodwin, A. J. Moseley, M. Q. Kearley, R. C. Morris, C. J. G. Kirkby, J. Thompson, R. C. Goodfellow, and I. Bennion, "Optoelectronic component arrays for optical interconnection of cjrcuits and subsystems," IEEE J. Lightwave Technol., vol. 9, pp. 1639-1645, Dec. 1991.

[6] K. W. Goossen, J. A. Walker, L. A. D'Asaro, S. P. Hui, and B. Tseng, "GaAs MQW modulators integrated with silicon CMOS," IEEE Photon. Technol. Lett., vol. 7, pp. 360-362, 1995.

[7] AT\&T/ARPA Hybrid-SEED Workshop Technical Notes, July 1995 , unpublished.

[8] D. R. Rolston, B. Robertson, H. S. Hinton, and D. V. Plant, "Analysis of a microchannel interconnect based on the clustering of smart-pixeldevice windows," Appl. Opt., vol. 35, pp. 1220-1233, 1996.

[9] T. K. Woodward, A. V. Krishnamoorthy, A. L. Lentine, K. W. Goossen, J. A. Walker, J. E. Cunningham, W. Y. Jan, L. A. D'Asaro, L. M. F. Chirovsky, S. P. Hui, B. Tseng, D. Kossives, D. Dahringer, and R. E. Leibenguth, "1-Gb/s two-beam transimpedance smart-pixel optical receivers made from hybrid GaAs MQW modulators bonded to $0.8-\mu \mathrm{m}$ silicon CMOS," IEEE Photon. Technol. Lett., vol. 8, pp. 422-424, 1996.

[10] F. B. McCormick, T. J. Cloonan, A. L. Lentine, J, M. Sasian, R. L. Morrison, M. G. Beckman, S. L. Walker, M. J. Wojcik, S. J, Hinterlong, R. J. Crisci, R. A. Novotny, and H. S. Hinton, "Fivestage free-space optical switching network with field-effect-transistor self-electro-optic-effect-device smart-pixel arrays," Appl. Opt., vol. 33 no. 8, pp. 1601-1618, Mar. 1994.

[11] S. M. Prince, F. A. P. Tooley, S. Wakelin, and M. R. Taghizadeh, "Implementation of optical perfect-shuffle module," Appl. Opt., vol. 34, no. 11 , pp. 1775-1782, Apr. 1994

[12] M. P. Y. Desmulliez, F. A. P. Tooley, J. A. B. Dines, N. L. Grant, D. J. Goodwill, D. Baillie, B. S. Wherrett, P. W. Foulk, S. Ashcroft, and P. Black, "Perfect-shuffle interconnected bitonic sorter: Optoelectronic design," Appl. Opt., vol. 34, no. 23, pp. 5077-5090, Aug. 1995.

[13] K. Hamanaka, "Optical bus interconnection system using Selfoc lenses," Opt. Lett., vol. 16, no. 16, pp. 1222-1224, Aug. 1991.

[14] S. Wakelin and M.W. Derstine, "Design and fabrication considerations for construction of monolithic hybrid optical components for optical computing applications," Optical Computing 1995i Tech. Dig. Ser. (Opt. Soc. Amer.), vol. 10, pp. 115-117, Mar. 1995.

[15] D. V. Plant, B. Robertson, H. S. Hinton, M. R. Ayliffe, G. C. Boisset, D. J. Goodwill, D. N. Kabal, R. Iyer, Y. S. Liu, ID. R. Rolston, W. M Robertson, and M. R. Taghizadeh, "A multistage CMOS-SEED optical backplane demonstration system," in Int. Top. Meet. Opt. Comput. 96, Sendai, Japan, Apr. 1996, pp. 14-15.

[16] F. A. P. Tooley, S. M. Prince, M. R. Taghizadeh, F. B. McCormick, M. W. Derstine, and S. Wakelin, "Implementation of a hybrid lens," Appl. Opt., vol. 34, no. 28, pp. 6471-6480, Oct. 1995.

[17] I. Redmond and E. Schenfeld, "Experimental results of a 64 channel, free-space optical interconnection network for massively parallel processing," Inst. Phys. Conf. Ser., no. 139, pt. II, pp. 149-152, 1995.

[18] H. J. White, G. M. Proudley, C. Stace, N. A. Brownjohn, A. C. Walker, M. R. Taghizadeh, B. Robertson, C. P. Barrett, W. A. Crossland, J. R. Brocklehurst, M. J. Birch, M. Snook, and D. Vass, "Development of an optical free-space crossbar," Inst. Phys. Conf. Ser, no. 139, pt. II, pp. 183-186, 1995

[19] F. B. McCormick, F. A. P. Tooley, T. J. Cloonan, J, M. Sasian, H. S. Hinton, K. O. Mersereau, and A. Y. Feldblum, "Optical interconnection using microlens arrays," Opt. Quantum Electron., vol. 24, pp S465-S477, 1992

[20] S. Matsuo, T. Nakahara, Y. Kohama, Y. Ohiso, S. Fukushima, and T. Kurokawa, "Monolithically integrated photonic switching device using an MSM PD, MESFET's, and a VCSEL," IEEE Photon. Technol. Lett., vol. 7, pp. 1165-1167, 1995 .

[21] D. V. Plant, B. Robertson, H. S. Hinton, M. H. Ayliffe, G. C. Boisset, W. Hsiao, D. Kabal, N. H. Kim, Y. S. Liu, M. R. Otazo, D. Paulasek, A. Z. Shang, J. Simmons, and W. M. Robertson, "A $4 \times 4$ VCSEL/MSM optical backplane demonstrator system," presented at the Annual LEOS Meeting, San Francisco, CA, Oct. 1995, paper PD2.4. 
[22] K. Zurl, E. Gluch, B. Schafer, J. Schwider, D. Fey, and W. Exhard, "Smart pixels with VCSELs: Potential and demonstration system," Inst. Phys. Conf. Ser., no. 139, pt. V, pp. 507-510, 1995.

[23] S. M. Fike, B. Buchanan, N. M. Jokerst, M. A. Brooke, T. G. Morris, and S. P. DeWeerth, " $8 \times 8$ array of thin-film photodetectors vertically" electrically interconnected to silicon circuitry," IEEE Photon. Technol. Lett., vol. 7, pp. 1168-1170, 1995.

[24] D. J. Goodwill, A. C. Walker, C. R. Stanley, M. C. Holland, and M. McElhinney, "Improvements in strain-balanced InGaAs/GaAs optical modulators for $1047 \mathrm{~nm}$ operation," Appl. Phys. Lett., vol. 64, no. 10, pp. 1192-1194, Mar. 1994.

[25] S. Haselbeck, H. Schreiber, J. Schwider and N. Streibl, "Microlenses fabricated by melting a photoresist on a base layer," Opt. Eng., vol. 32, no. 6, pp. 1322-1324, June 1993.

[26] Z.-L. Liau, D. W. Nam, and R. G. Waarts, "Tolerances in microlens fabrication by multilevel etching and mass-transport smoothing," Appl. Opt., vol. 33, no. 31, pp. 7371-7374, Nov. 1994.

[27] F. B. McCormick, Photonics in Switching, J. E. Midwinter, Ed. New York: Academic, 1993.

[28] D. J. Reiley, J. M. Sasian, and M. L. Beckman, "Optomechanical design of a robust free-space optical switching system," SPIE Photonics West, San Jose, Feb. 1996

[29] J. M. Miller, M. R. Taghizadeh, J. Turunen, N. Ross, E. Noponen, and A. Vasara, "Kinoform array illuminators in fused silica," J. Modern Opt., vol. 40, no. 4, pp. 723-732, 1993.

[30] S. J. Walker, J. Jahns, L. Li, W. M. Mansfield, P. Mulgrew, D. M Tennant, C. W. Roberts, L. C. West, and N. K. Ailawadi, "Design and fabrication of high-efficiency beam splitters and beam deflector for integrated planar micro-systems," Appl. Opt., vol. 32, no. 14, pp. 2494-2501, May 1993.

[31] D. V. Plant, B. Robertson, H. S. Hinton, W. M. Robertson, G. C. Boisset, N. H. Kim, Y. S. Liu, M. R. Otazo, D. R. Rolston, and A. Z. Shang, "An optical backplane demonstrator system based on FET-SEED smart pixel arrays and diffractive lenslet arrays," IEEE Photon. Technol. Lett. vol. 7, pp. 1057-1059, Sept. 1995

[32] P. Belland and J. P. Crenn, "Changes in the characteristics of a Gaussian beam weakly diffracted by a circular aperture," Appl. Opt., vol. 21, no. 3, pp. 522-527, Feb. 1992.

[33] J. Moisel and K.-H. Brenner, "A universal module for split-andjoin operations by cascading refractive microoptical elements," Optical Computing 1995 Tech. Dig. Ser. (Opt. Soc. Amer.), vol. 10, pp. 118-120, Mar. 1995.
Dominic J. Goodwill was born in London, U.K. He received the Ph.D. degree from Heriot-Watt University, U.K., in 1991 for work on nonlinearities in semiconductor waveguides.

He remained at Heriot-Watt and developed III-V modulator arrays for optical computing applications. He then joined the group of Professor Hinton at the University of Colorado at Boulder, where he designed optics and mechanics for free-space optical interconnections in backplanes. His present research at the University of Colorado is on the packaging of laser arrays and fiber ribbons for parallel optical data links, involving tapered polymer waveguides and flip-chip solder bonding.

Kent E. Devenport was born in Kirkwood, MO, in 1972. He received the B.S degree in electrical engineering from the University of Missouri-Columbia in 1994, graduating summa cum laude, and the M.S. degree in electrical engineering from the University of Colorado-Boulder in 1996.

He has also worked as an intern at Bell Laboratories of Lucent Technologies, Holmdel, NJ. His research interests include VLSI design, optoelectronic integration techniques, and optical backplane architectures.

H. Scott Hinton (S'81-M'82-SM'92), for photograph and biography, see this issue, p. 23. 